\title{
PROPERTIES OF FIXED POINT SET OF A MULTIVALUED MAP
}

\author{
ABDUL RAHIM KHAN
}

Received 15 September 2004 and in revised form 21 November 2004

Properties of the set of fixed points of some discontinuous multivalued maps in a strictly convex Banach space are studied; in particular, affirmative answers are provided to the questions related to set of fixed points and posed by Ko in 1972 and $\mathrm{Xu}$ and Beg in 1998. A result regarding the existence of best approximation is derived.

\section{Introduction}

The study of fixed points for multivalued contractions and nonexpansive maps using the Hausdorff metric was initiated by Markin [17]. Later, an interesting and rich fixed point theory for such maps has been developed. The theory of multivalued maps has applications in control theory, convex optimization, differential inclusions, and economics (see, e.g., $[3,8,16,22])$.

The theory of multivalued nonexpansive mappings is harder than the corresponding theory of single-valued nonexpansive mappings. It is natural to expect that the theory of nonself-multivalued noncontinuous functions would be much more complicated.

The concept of a $*$-nonexpansive multivalued map has been introduced and studied by Husain and Latif [9] which is a generalization of the usual notion of nonexpansiveness for single-valued maps. In general, *-nonexpansive multivalued maps are neither nonexpansive nor continuous (see Example 3.7).

$\mathrm{Xu}$ [22] has established some fixed point theorems while Beg et al. [2] have recently studied the interplay between best approximation and fixed point results for $*$-nonexpansive maps defined on certain subsets of a Hilbert space and Banach space. For this class of functions, approximating sequences to a fixed point in Hilbert spaces are constructed by Hussain and Khan [10] and its applications to random fixed points and best approximations in Fréchet spaces are given by Khan and Hussain [12].

In this paper, using the best approximation operator, we (i) establish certain properties of the set of fixed points of a $*$-nonexpansive multivalued nonself-map in the setup of a strictly convex Banach space, (ii) prove fixed point results for $*$-nonexpansive random maps in a Banach space under several boundary conditions, and (iii) provide affirmative answers to the questions posed by Ko [14] and $\mathrm{Xu}$ and Beg [24] related to the set of fixed points. 


\section{Notations and preliminaries}

Let $C$ be a subset of a normed space $X$. We denote by $2^{X}, C(X), K(X), C C(X), C K(X)$, and $C B(X)$ the families of all nonempty, nonempty closed, nonempty compact, nonempty closed convex, nonempty convex compact, and nonempty closed bounded subsets of $X$, respectively.

Define $d(x, C)=\inf _{y \in C} d(x, y)$. The Hausdorff metric on $C B(X)$ induced by the metric $d$ on $X$ is denoted by $H$ and is defined as

$$
H(A, B)=\max \left\{\sup _{a \in A} d(a, B), \sup _{b \in B} d(b, A)\right\} .
$$

A mapping $T: C \rightarrow C B(X)$ is a contraction if for any $x, y \in C, H(T x, T y) \leq k d(x, y)$, where $0 \leq k<1$. If $k=1$, then $T$ is called a nonexpansive map. If $H(T x, T y)<d(x, y)$ whenever $x \neq y$ in $C$, then $T$ is called a strictly nonexpansive mapping [14].

A multivalued map $T: C \rightarrow 2^{X}$ is said to be

(i) *-nonexpansive if for all $x, y \in C$ and $u_{x} \in T x$ with $d\left(x, u_{x}\right)=d(x, T x)$, there exists $u_{y} \in T y$ with $d\left(y, u_{y}\right)=d(y, T y)$ such that $d\left(u_{x}, u_{y}\right) \leq d(x, y)$ (see $\left.[9,10]\right)$,

(ii) strictly $*$-nonexpansive if for all $x \neq y$ in $C$ and $u_{x} \in T x$ with $d\left(x, u_{x}\right)=d(x, T x)$, there exists $u_{y} \in T y$ with $d\left(y, u_{y}\right)=d(y, T y)$ such that $d\left(u_{x}, u_{y}\right)<d(x, y)$,

(iii ) upper semicontinuous (usc) (lower semicontinuous (lsc)) if $T^{-1}(B)=\{x \in C$ : $T x \cap B \neq \phi\}$ is closed (open) for each closed (open) subset $B$ of $X$. If $T$ is both usc and lsc, then $T$ is continuous,

(iv) asymptotically contractive [19] if there exist some $c \in(0,1)$ and $r>0$ such that

$$
\|y\| \leq c\|x\|, \quad \forall y \in T x, \forall x \in C \backslash r B_{X},
$$

where $B_{X}$ is the closed unit ball of $X$.

The map $T: C \rightarrow C B(X)$ is called (i) $H$-continuous (continuous with respect to Hausdorff metric $H)$ if and only if for any sequence $\left\{x_{n}\right\}$ in $C$ with $x_{n} \rightarrow x$, we have $H\left(T x_{n}, T x\right)$ $\rightarrow 0$ (the two concepts of set-valued continuity are equivalent when $T$ is compact-valued (cf. [8, Theorem 20.3, page 94])); (ii) demiclosed at 0 if the conditions $x_{n} \in C, x_{n}$ converges weakly to $x, y_{n} \in T x_{n}$ and $y_{n} \rightarrow 0$ imply that $0 \in T x$. An element $x$ in $C$ is called a fixed point of a multivalued map $T$ if and only if $x \in T x$. The set of all fixed points of $T$ will be denoted by $F(T)$.

For each $x \in X$, let $P_{C}(x)=\{z \in C: d(x, z)=d(x, C)\}$. Any $z \in P_{C}(x)$ is called a point of best approximation to $x$ from $C$. If $P_{C}(x) \neq \phi$ (singleton) for each $x \in X$, then $C$ is called a proximinal (Chebyshev) set, respectively. If $C$ is proximinal, then the mapping $P_{C}: X \rightarrow 2^{C}$ is well defined and is called the metric projection.

The space $X$ is said to have the Oshman property (see [18]) if it is reflexive and the metric projection on every closed convex subset is usc.

For the multivalued map $T$ and each $x \in C$, we follow Xu [22] to define the best approximation operator, $P_{T}(x)=\left\{u_{x} \in T x: d\left(x, u_{x}\right)=d(x, T x)\right\}$, (possibly empty set).

A single-valued (multivalued) map $f: C \rightarrow X\left(F: C \rightarrow 2^{X}\right)$ is said to be a selector of $T$ if $f(x) \in T x(F x \subseteq T x)$, respectively, for each $x \in C$. 
The space $X$ is said to have the Opial condition if for every sequence $\left\{x_{n}\right\}$ in $X$ weakly convergent to $x \in X$, the inequality

$$
\liminf _{n \rightarrow \infty}\left\|x_{n}-x\right\|<\liminf _{n \rightarrow \infty}\left\|x_{n}-y\right\|
$$

holds for all $y \neq x$.

Every Hilbert space and the spaces $\ell_{p}(1 \leq p<\infty)$ satisfy the Opial condition.

The inward set $I_{C}(x)$ of $C$ at $x \in X$ is defined by $I_{C}(x)=\{x+\gamma(y-x): y \in C$ and $\gamma>0\}$. We will denote the closure of $C$ by $\mathrm{cl}(C)$.

Let $(\Omega, \mathscr{A})$ denote a measurable space with $\mathscr{A}$ sigma algebra of subsets of $\Omega$. A mapping $T: \Omega \rightarrow 2^{X}$ is called measurable if for any open subset $B$ of $X, T^{-1}(B)=\{\omega \in \Omega: T(\omega) \cap$ $B \neq \phi\} \in \mathscr{A}$. A mapping $\zeta: \Omega \rightarrow X$ is said to be a measurable selector of a measurable mapping $T: \Omega \rightarrow 2^{X}$ if $\zeta$ is measurable and for any $\omega \in \Omega, \zeta(\omega) \in T(\omega)$. A mapping $T: \Omega \times C \rightarrow 2^{X}$ is a random operator if for any $x \in C, T(\cdot, x)$ is measurable. A mapping $\zeta: \Omega \rightarrow C$ is said to be a random fixed point of $T$ if $\zeta$ is a measurable map such that for every $\omega \in \Omega, \zeta(\omega) \in T(\omega, \zeta(\omega))$.

A random operator $T: \Omega \times C \rightarrow 2^{X}$ is said to be continuous (nonexpansive, $*$-nonexpansive, convex, etc.) if for each $\omega \in \Omega, T(\omega, \cdot)$ is continuous (nonexpansive, *-nonexpansive, convex, etc.).

The following results are needed.

Proposition 2.1 (see [3, Proposition 2.2]). Let E be a metric space. If $T: \Omega \rightarrow C(E)$ is a multivalued mapping, then the following conditions are equivalent:

(i) $T$ is measurable;

(ii) $\omega \rightarrow d(x, T(\omega))$ is a measurable function of $\omega$ for each $x \in E$;

(iii) there exists a sequence $\left\{f_{n}(\omega)\right\}$ of measurable selectors of $T$ such that $\operatorname{cl}\left\{f_{n}(\omega)\right\}=$ $T(\omega)$ for all $\omega$ in $\Omega$.

Theorem 2.2 (see [24, Theorem 3.1]). Let $C$ be a nonempty separable weakly compact convex subset of a Banach space $X$. Suppose that the map $T: \Omega \times C \rightarrow K(C)$ is a nonexpansive random mapping. If for each $\omega \in \Omega, I-T(\omega, \cdot)$ is demiclosed at 0 , then the fixed point set function $F$ of $T$ given by $F(\omega)=\{x \in C: x \in T(\omega, x)\}$ is measurable (and hence $T$ has a random fixed point).

\section{3. *-nonexpansive maps}

The properties of the set of fixed points of single-valued and multivalued maps have been considered by a number of authors (see, e.g., Agarwal and O’Regan [1], Browder [4], Bruck [5], Espínola et al. [6], Ko [14], Schöneberg [20], and Xu and Beg [24]). For a wide class of unbounded closed convex sets $C$ in a Banach space, there exist nonexpansive maps $T: C \rightarrow K(C)$ which fail to have a fixed point (see [13]).

We obtain some properties of the set of fixed points of a $*$-nonexpansive map on a Banach space with values which are not necessarily subsets of the domain.

Markin [17], Xu [22], and Jachymski [11] have utilized "selections;" we employ "nonexpansive selector," $P_{T}$, of a $*$-nonexpansive map $T$ to study the structure of the set of 
fixed points of $T$. Consequently, we obtain generalized and improved versions of many results in the current literature.

In Theorem 8.2, Browder [4] has established the following result.

Theorem 3.1. Let $C$ be a nonempty closed, convex, subset of a strictly convex Banach space $X$ and let $T: C \rightarrow C$ be a nonexpansive map. Then the set $F(T)$ of fixed points of $T$ is closed and convex.

Ko [14] pointed out that Theorem 3.1 need not hold for multivalued nonexpansive mappings as follows.

Example 3.2 (see [14, Example 3]). Consider $C=[0,1] \times[0,1]$ with the usual norm. Define $T: C \rightarrow C K(C)$ by

$$
T(x, y)=\text { the triangle with vertices }(0,0),(x, 0) \text {, and }(0, y) \text {. }
$$

Note that $T$ is nonexpansive and the norm in $\mathbb{R}^{2}$ is strictly convex. But the set $F(T)=$ $\{(x, y):(x, y) \in C$ and $x y=0\}$ is not convex.

The following generalization of Theorem 3.1 for $*$-nonexpansive continuous mappings is obtained in [15].

Theorem 3.3. Let $X$ be a strictly convex Banach space and $C$ a nonempty weakly compact convex subset of $X$. Let $T: C \rightarrow C C(C)$ be a $*$-nonexpansive map such that $F(T)$ is nonempty. Then the set $F(T)$ is convex and is closed if $T$ is continuous.

We present a new proof, through the best approximation operator, of Theorem 3.3 without assuming any type of continuity of the map $T$ and obtain the following structure theorem.

Theorem 3.4. Let $X$ be a strictly convex Banach space and $C$ a nonempty weakly compact convex subset of $X$. Let $T: C \rightarrow C C(C)$ be a *-nonexpansive map such that $F(T)$ is nonempty. Then the set $F(T)$ is closed and convex.

Proof. For each $x \in C$, its image $T x$ is weakly compact and convex and thus each $T x$ is Chebyshev. Hence, each $u_{x}$ in $P_{T}(x)$ is unique. Thus by the definition of $*$-nonexpansiveness of $T$, there is $u_{y}=P_{T}(y) \in T y$ for all $y$ in $C$ such that

$$
\left\|P_{T}(x)-P_{T}(y)\right\|=\left\|u_{x}-u_{y}\right\| \leq\|x-y\| .
$$

Hence, $P_{T}: C \rightarrow C$ is a nonexpansive selector of $T$ (see also [22]). By the definition of $P_{T}$, we have for each $y \in C$,

$$
d\left(y, P_{T}(y)\right)=d\left(y, u_{y}\right)=d(y, T y)
$$

Equation (3.3) now implies that $F(T)=F\left(P_{T}\right)$. Thus $F\left(P_{T}\right)$ and hence $F(T)$ is closed and convex by Theorem 3.1 .

The following example illustrates our results. 
Example 3.5. Let $T:[0,1] \rightarrow 2^{[0,1]}$ be a multivalued map defined by

$$
T x= \begin{cases}{\left[0, \frac{1}{2}\right],} & x \neq \frac{1}{2}, \\ {[0,1],} & x=\frac{1}{2} .\end{cases}
$$

Then

$$
P_{T}(x)= \begin{cases}x, & x \in\left[0, \frac{1}{2}\right], \\ \frac{1}{2}, & x \in\left(\frac{1}{2}, 1\right] .\end{cases}
$$

This implies that $T$ is a $*$-nonexpansive map. Further, $T$ is usc but not lsc (see [8, Remark 15.2, page 71]) and hence $T$ is not continuous according to both definitions as $T$ is compact-valued. Note that $F(T)=[0,1 / 2]$ is closed and convex.

If $T$ is a single-valued strictly nonexpansive map, then $F(T)$ is a singleton. In general, this is not true for a multivalued nonexpansive map [17]. The set $F(T)$ is said to be singleton in a generalized sense if there exists $x \in F(T)$ such that $F(T) \subseteq T x$. Ko has given an example of a strictly nonexpansive mapping $T: C \rightarrow C C(C)$, in a strictly convex Banach space, for which the set $F(T)$ is not singleton in a generalized sense (cf. [17, Example 4]). Ko raised the following question: is $F(T)$ singleton in a generalized sense if $T$ is nonexpansive, $I$ is the identity operator, and $I-T$ is convex?

The following proposition provides an affirmative answer to this question for strictly *-nonexpansive multivalued mappings.

Proposition 3.6. Let $C$ be a nonempty closed convex subset of a reflexive strictly convex Banach space $X$ and let $T: C \rightarrow C C(C)$ be a strictly $*$-nonexpansive map such that $F(T)$ is nonempty. Then the set $F(T)$ is singleton in a generalized sense.

Proof. Any closed convex subset of a reflexive strictly convex Banach space is Chebyshev, so each $T x$ is Chebyshev. Thus as in the proof of Theorem 3.4, $P_{T}: C \rightarrow C$ is a strictly nonexpansive selector of $T$ satisfying (3.3). Hence, $F(T)=F\left(P_{T}\right)$ is singleton in a generalized sense as required.

The following example supports the above proposition.

Example 3.7. Let $T:[0,1] \rightarrow 2^{[0,1]}$ be a multivalued map defined by

$$
T x= \begin{cases}\left\{\frac{1}{2}\right\}, & x \in\left[0, \frac{1}{2}\right) \cup\left(\frac{1}{2}, 1\right], \\ {\left[\frac{1}{4}, \frac{3}{4}\right],} & x=\frac{1}{2} .\end{cases}
$$


Then $P_{T}(x)=\{1 / 2\}$ for every $x \in[0,1]$. This implies that $T$ is a strictly $*$-nonexpansive map:

$$
\begin{aligned}
H\left(T\left(\frac{1}{3}\right), T\left(\frac{1}{2}\right)\right) & =H\left(\left\{\frac{1}{2}\right\},\left[\frac{1}{4}, \frac{3}{4}\right]\right) \\
& =\max \left\{\sup _{a \in\{1 / 2\}} d\left(a,\left[\frac{1}{4}, \frac{3}{4}\right]\right), \sup _{b \in[1 / 4,3 / 4]} d\left(b, \frac{1}{2}\right)\right\} \\
& =\max \left\{0, \frac{1}{4}\right\}=\frac{1}{4}>\frac{1}{6}=\left|\frac{1}{3}-\frac{1}{2}\right| .
\end{aligned}
$$

This implies that $T$ is not nonexpansive. Obviously, $T$ is compact-valued. Next we show that $T$ is not lsc.

Let $V_{1 / 4}$ be any small open neighbourhood of $1 / 4$. Then the set

$$
T^{-1}\left(V_{1 / 4}\right)=\left\{x \in[0,1]: T x \cap V_{1 / 4} \neq \phi\right\}=\left\{\frac{1}{2}\right\}
$$

is not open. Thus $T$ is not continuous in the sense of both definitions.

Note that $F(T)=\{1 / 2\}$ is singleton in a generalized sense.

The conclusion of Proposition 3.6 does not hold for $*$-nonexpansive maps as follows. Example 3.8. Let $C=[0, \infty)$ and $T: C \rightarrow C K(C)$ be defined by

$$
T x=[x, 2 x] \quad \text { for } x \in C .
$$

Then $P_{T}(x)=\{x\}$ for every $x \in C$. This clearly implies that $T$ is $*$-nonexpansive but not nonexpansive (cf. [22]). Note that $F(T)=C$ and there does not exist any $x$ in $F(T)$ such that $F(T) \subseteq T x$. Thus $F(T)$ is not singleton in a generalized sense.

The above example also indicates that the fixed point set of a $*$-nonexpansive map need not be bounded in general. However, if $T$ is asymptotically contractive, then we have the following affirmative result.

Theorem 3.9. Let $X$ be a uniformly convex Banach space and $C$ a nonempty closed convex subset of $X$. Let $T: C \rightarrow C C(C)$ be a *-nonexpansive map which is asymptotically contractive on $C$. Then $F(T)$ is nonempty closed, convex, and bounded.

Proof. The map $T$ has a nonexpansive selector $f$ which is also asymptotically contractive by the asymptotic contractivity of T. Further, $F(T)=F(f)$ is nonempty closed bounded and convex (see [19, Corollary 3 and Remark (a)]).

We are now ready to derive a version of the Ky-Fan best approximation theorem [7] (compare the result with [10, Theorem 3.1] and [21, Theorem 4.3]).

THEOREM 3.10. Let $C$ be a nonempty closed convex subset of a strictly convex Banach space $X$ with the Oshman property. If $T: C \rightarrow C C(X)$ is an $H$-continuous (or a $*$-nonexpansive) map and $T(C)$ is relatively compact, then there exists $y \in C$ such that

$$
d(y, T y)=\|y-f y\|=d\left(f y, \operatorname{cl}\left(I_{C}(y)\right)\right), \quad \text { for some continuous selector } f \text { of } T \text {. }
$$


Proof. The Hausdorff continuity of $T$ implies that $f=P_{T}: C \rightarrow X$ is a continuous selector of $T$. Since $T(C)$ is relatively compact and $f(C) \subseteq T(C)$, therefore $f(C)$ is relatively compact. By $[18$, Theorem 3(0)] and (3.3), we obtain

$$
d(y, T y)=d(y, f y)=d\left(f y, \operatorname{cl}\left(I_{C}(y)\right)\right), \quad \text { for some } y \in C .
$$

The proof for $*$-nonexpansive map is similar.

As an application of Theorems 3.4 and 3.10, we obtain the following extension of [9, Theorem 3.2], [22, Corollary 1], and Theorem 3.3.

Corollary 3.11. Let $C$ be a nonempty closed convex subset of a strictly convex Banach space $X$ with the Oshman property. If $T: C \rightarrow C C(C)$ is a *-nonexpansive map and $T(C)$ is relatively compact, then $F(T)$ is nonempty closed and convex.

Remark 3.12. The map $T$ in Example 3.2 is neither $*$-nonexpansive nor has a nonexpansive selection $f$ with $F(f)=F(T)$; for if $T$ is so, then using the same argument as in the proof of Theorem 3.4, $T$ should have a nonexpansive selector $P_{T}: C \rightarrow C$ such that $F(T)=F\left(P_{T}\right)$, which should be convex by Theorem 3.1; a contradiction.

$\mathrm{Xu}$ [23] obtained the randomization of a remarkable fixed point theorem for multivalued nonexpansive maps due to Lim [16]. Further, $\mathrm{Xu}$ and Beg stated that it is unknown whether the fixed point set function $F$ in this case is measurable (see [24, page $69]$ ). We prove that the fixed point set function $F$ is measurable if the underlying map is *-nonexpansive.

Theorem 3.13. Let $C$ be a nonempty separable closed bounded convex subset of a Banach space $X$ and $T: \Omega \times C \rightarrow K(C) a *$-nonexpansive random operator. Then the fixed point set function $F$ of $T$ given by $F(\omega)=\{x \in C: x \in T(\omega, x)\}$ is measurable (and hence $T$ has a random fixed point) provided one of the following conditions holds:

(i) $T(\omega, \cdot)$ is convex for each $\omega \in \Omega$ and $X$ is a uniformly convex space,

(ii) $C$ is weakly compact and $I-T$ is demiclosed at 0 ,

(iii) $C$ is weakly compact and $X$ satisfies the Opial condition.

Proof. (i) As before, for each $\omega \in \Omega, P_{T}(\omega, \cdot): \Omega \times C \rightarrow C$ is a nonexpansive selector of $T(\omega, \cdot)$ and for each $y \in C, \omega \in \Omega$,

$$
d\left(y, P_{T}(\omega, y)\right)=d\left(y, u_{y}\right)=d(y, T(\omega, y))
$$

By Proposition 2.1, $T(\cdot, x)$ is measurable if and only if for each $x$ in $C$, the function $d(x, T(\cdot, x))$ is measurable. Thus by $(3.12)$, for each $x$ in $C, d\left(x, P_{T}(\cdot, x)\right)$ is measurable and hence again by Proposition 2.1, $P_{T}(\cdot, x)$ is measurable (see also [12, Proposition 3.6]). Thus $P_{T}: \Omega \times C \rightarrow C$ is a nonexpansive random operator.

We observe that if $X$ is a uniformly convex space, then $I-P_{T}(\omega, \cdot)$ is demiclosed. Also (3.12) implies that fixed point set function $G$ of $P_{T}$ given by $G(\omega)=\left\{x \in C: x=P_{T}(\omega, x)\right\}$ is equal to $F(\omega)=\{x \in C: x \in T(\omega, x)\}$ for each $\omega \in \Omega$. Consequently, $G$, and hence $F$, is measurable by Theorem 2.2 . 
(ii) Note that $P_{T}(\omega, \cdot): C \rightarrow K(C)$ is a nonexpansive selector of $T(\omega, \cdot)$. Also for each $y \in C, \omega \in \Omega$,

$$
d\left(y, P_{T}(\omega, y)\right) \leq d\left(y, u_{y}\right)=d(y, T(\omega, y)) \leq d\left(y, P_{T}(\omega, y)\right)
$$

The measurability of $P_{T}$ follows from the arguments adopted in part (i) using (3.13) instead of (3.12). The demiclosedness of $I-T(\omega, \cdot)$ at 0 implies that $I-P_{T}(\omega, \cdot)$ is also demiclosed at 0 as follows.

Suppose that $x_{n} \rightarrow x_{0}$ weakly and $y_{n} \in I-P_{T}\left(\omega, x_{n}\right)$ with $y_{n} \rightarrow 0$ strongly. Note that $y_{n} \in I-P_{T}\left(\omega, x_{n}\right) \subseteq I-T\left(\omega, x_{n}\right)$ and $I-T(\omega, \cdot)$ is demiclosed at 0 so $0 \in I-T\left(\omega, x_{0}\right)$ for each $\omega \in \Omega$. This implies that $x_{0} \in T\left(\omega, x_{0}\right)$ and hence $0=d\left(x_{0}, T\left(\omega, x_{0}\right)\right)=d\left(x_{0}, P_{T}\left(\omega, x_{0}\right)\right)$ for each $\omega \in \Omega$. Thus $x_{0} \in P_{T}\left(\omega, x_{0}\right)$ implies that $I-P_{T}(\omega, \cdot)$ is demiclosed at 0 for each $\omega \in \Omega$. Thus $G$, and hence, $F$ is measurable by Theorem 2.2 .

(iii) It is well known that if $C$ is a weakly compact subset of a Banach space $X$ satisfying the Opial condition and $f: C \rightarrow K(C)$ is nonexpansive, then $I-f$ is demiclosed on $C$. Hence, $I-P_{T}(\omega, \cdot)$ is demiclosed for each $\omega \in \Omega$ and the conclusion now follows from part (ii).

Remark 3.14. It is not common at all that a nonexpansive multivalued mapping admits a single-valued nonexpansive selection (cf. Example 3.2 and Remark 3.12). However, in the general setup of metric linear spaces, $*$-nonexpansive maps have nonexpansive selector satisfying a very useful relation (3.3).

\section{Acknowledgment}

The author gratefully acknowledges the support provided by King Fahd University of Petroleum \& Minerals during this research.

\section{References}

[1] R. P. Agarwal and D. O'Regan, On the topological structure of fixed point sets for abstract Volterra operators on Fréchet spaces, J. Nonlinear Convex Anal. 1 (2000), no. 3, 271-286.

[2] I. Beg, A. R. Khan, and N. Hussain, Approximation of*-nonexpansive random multivalued operators on Banach spaces, J. Aust. Math. Soc. 76 (2004), no. 1, 51-66.

[3] T. D. Benavides, G. L. Acedo, and H. K. Xu, Random fixed points of set-valued operators, Proc. Amer. Math. Soc. 124 (1996), no. 3, 831-838.

[4] F. E. Browder, Nonlinear operators and nonlinear equations of evolution in Banach spaces, Nonlinear Functional Analysis (Proc. Sympos. Pure Math., Vol. XVIII, Part 2, Chicago, Ill., 1968), American Mathematical Society, Rhode Island, 1976, pp. 1-308.

[5] R. E. Bruck Jr., Properties of fixed-point sets of nonexpansive mappings in Banach spaces, Trans. Amer. Math. Soc. 179 (1973), 251-262.

[6] R. Espínola, E. S. Kim, and W. A. Kirk, Fixed point properties of mappings satisfying local contractive conditions, Nonlinear Anal. Forum 6 (2001), no. 1, 103-111.

[7] K. Fan, Extensions of two fixed point theorems of F. E. Browder, Math. Z. 112 (1969), 234-240.

[8] L. Górniewicz, Topological Fixed Point Theory of Multivalued Mappings, Mathematics and Its Applications, vol. 495, Kluwer Academic Publishers, Dordrecht, 1999.

[9] T. Husain and A. Latif, Fixed points of multivalued nonexpansive maps, Math. Japon. 33 (1988), no. 3, 385-391. 
[10] N. Hussain and A. R. Khan, Applications of the best approximation operator to *-nonexpansive maps in Hilbert spaces, Numer. Funct. Anal. Optim. 24 (2003), no. 3-4, 327-338.

[11] J. R. Jachymski, Caristi's fixed point theorem and selections of set-valued contractions, J. Math. Anal. Appl. 227 (1998), no. 1, 55-67.

[12] A. R. Khan and N. Hussain, Random coincidence point theorem in Fréchet spaces with applications, Stochastic Anal. Appl. 22 (2004), no. 1, 155-167.

[13] W. A. Kirk and W. O. Ray, Fixed-point theorems for mappings defined on unbounded sets in Banach spaces, Studia Math. 64 (1979), no. 2, 127-138.

[14] H. M. Ko, Fixed point theorems for point-to-set mappings and the set of fixed points, Pacific J. Math. 42 (1972), 369-379.

[15] A. Latif, A. Bano, and A. R. Khan, Some results on multivalued s-nonexpansive maps, Rad. Mat. 10 (2001), no. 2, 195-201.

[16] T. C. Lim, A fixed point theorem for multivalued nonexpansive mappings in a uniformly convex Banach space, Bull. Amer. Math. Soc. 80 (1974), 1123-1126.

[17] J. T. Markin, Continuous dependence of fixed point sets, Proc. Amer. Math. Soc. 38 (1973), 545547.

[18] S. Park, Best approximations, inward sets, and fixed points, Progress in Approximation Theory, Academic Press, Massachusetts, 1991, pp. 711-719.

[19] J.-P. Penot, A fixed-point theorem for asymptotically contractive mappings, Proc. Amer. Math. Soc. 131 (2003), no. 8, 2371-2377.

[20] R. Schöneberg, A note on connection properties of fixed point sets of nonexpansive mappings, Math. Nachr. 83 (1978), 247-253.

[21] K.-K. Tan and X.-Z. Yuan, Random fixed point theorems and approximation, Stochastic Anal. Appl. 15 (1997), no. 1, 103-123.

[22] H. K. Xu, On weakly nonexpansive and * -nonexpansive multivalued mappings, Math. Japon. 36 (1991), no. 3, 441-445.

[23] A random fixed point theorem for multivalued nonexpansive operators in uniformly convex Banach spaces, Proc. Amer. Math. Soc. 117 (1993), no. 4, 1089-1092.

[24] H. K. Xu and I. Beg, Measurability of fixed point sets of multivalued random operators, J. Math. Anal. Appl. 225 (1998), no. 1, 62-72.

Abdul Rahim Khan: Department of Mathematical Sciences, King Fahd University of Petroleum \& Minerals, Dhahran 31261, Saudi Arabia

E-mail address: arahim@kfupm.edu.sa 


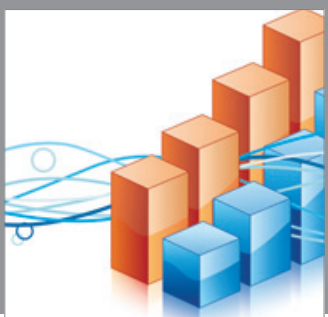

Advances in

Operations Research

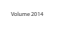

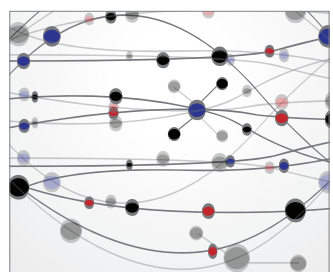

\section{The Scientific} World Journal
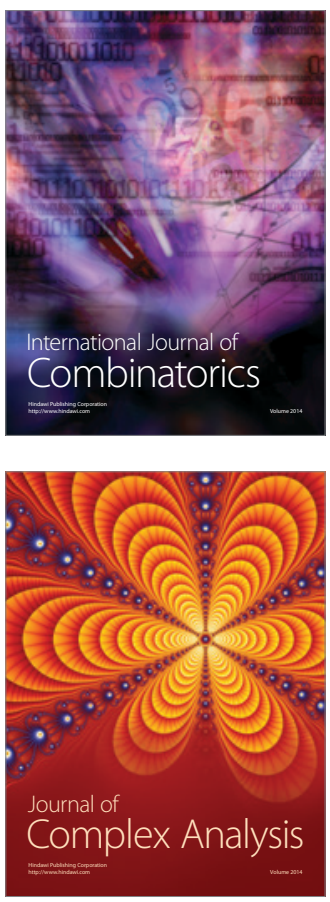

International Journal of

Mathematics and

Mathematical

Sciences
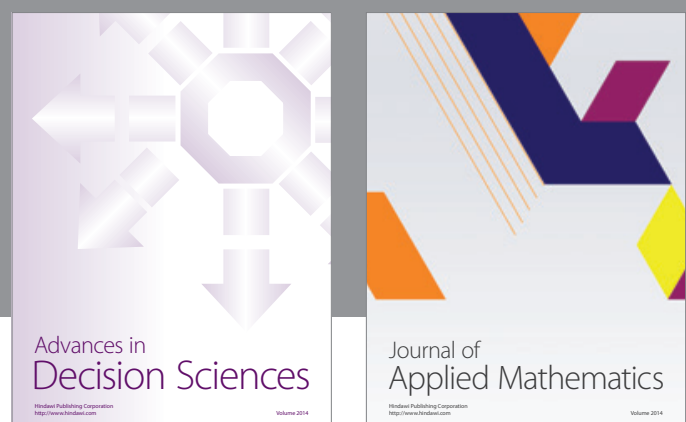

Journal of

Applied Mathematics
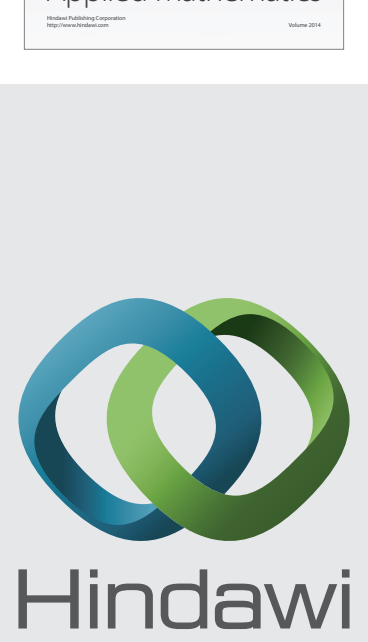

Submit your manuscripts at http://www.hindawi.com
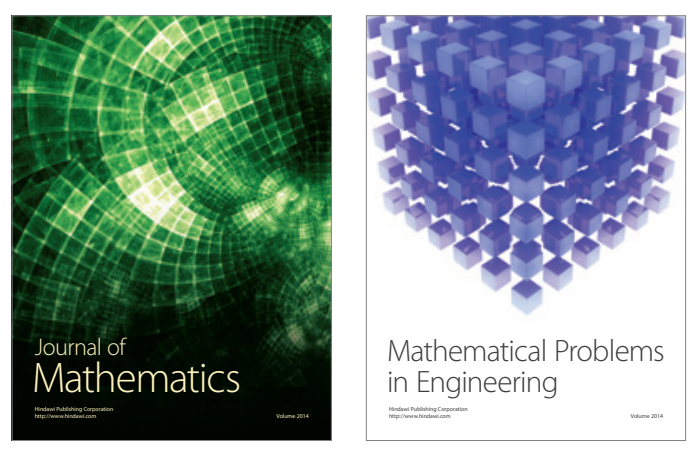

Mathematical Problems in Engineering
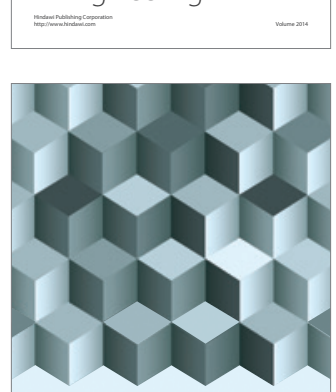

Journal of

Function Spaces
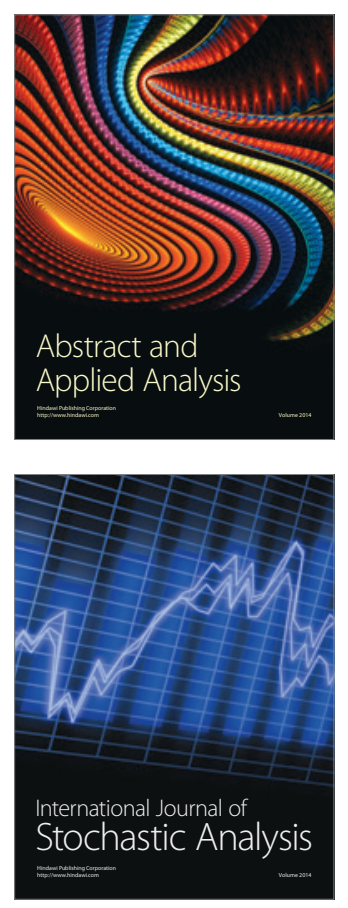

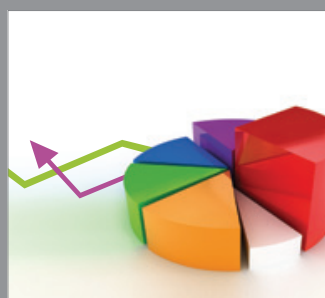

ournal of

Probability and Statistics

Promensencen
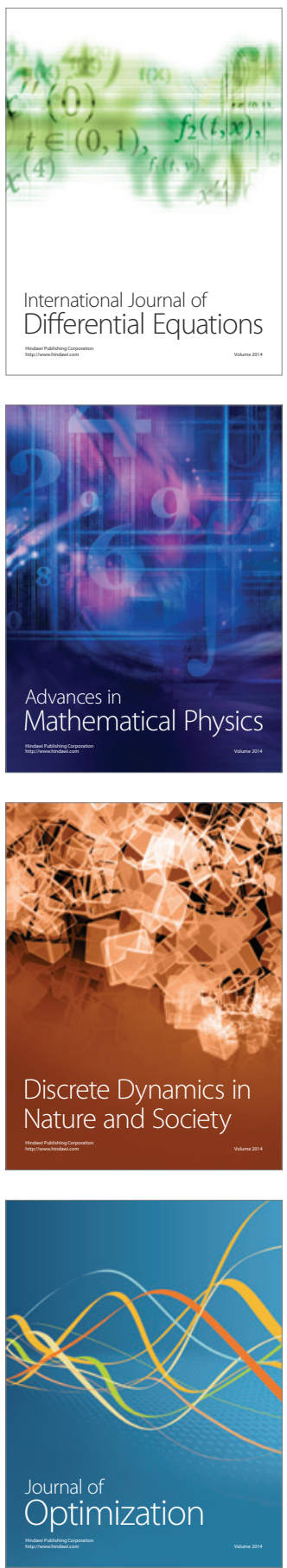